étage, limitation des porte-à-faux, etc.), permet de tout oser sans risque ni frais. Une nouvelle architecture doit donc nattre, dont le caractère sera une extrême fantaisie; l'enfantement de cette révolution demande quelque temps et surtout quelques hommes d'une certaine envergure.

\section{L'Invention du four électrique}

Le frix Nobel, la mort de Moissan, ainsi que l'affaire Lemoine, ont rappelé l'attention sur le four électrique, et la plupart de ceux que ces questions préoccupent attribucnt lo four electrique à Moissan. La gloire de ce dervier est assez grande pour n'avoir rien à craindre du rétablissement de la verité et il est permis de rendre le four clectri. que à celui qui l'a imaginé il y a vingt-six ans.

C'est en 1881 que $M$. Louis Clerc, aujourd'hui chef de l'exploitation du secteur Edison, poursuivant ses études sur la lampe Soleil dont il était l'inventeur, imagina le four électrique sous la forme même où Moissan l'employa quelques annees plus tard. Le brevet belge du 9 juillet 1881 , pris au nom do la Compagile belge de Lumière Electrique, $\dot{a}$ laquolle appartenait alors $M$. Clerc, montre la similitude des doux appareils, et c'est pour cette raison que nous le reprodusons ci-dessous, avec les figures qui l'accompagnaient. Cette similitude n'implique, d'ailleurs, nullement que Molssan ait eu connaissance alor's de cette antériorité; on 1896. faisant des expériences avec le four électrique à l'usine Trudaine, Moissan reconnut devant M. Clerc l'antélor'lté óvidente du brevet de 1881, la seule qu'il acceptat.

Le lour olectrique venait trop tôt en 1881 ; les ressources de l'industrie étaient insulfisantes pour l'exploitation utile de ses remarquables propriétés; cependant, avec le faible courunt doni il disposait, 15 amperes sous 160 volts, M. Clos e put obtenir la transformation du charbon en graphitc, et il reconnut que rien ne pouvait résister à la chaleur de lare. La conséquence en fut l'abandon de la lampe Soleil, faute d'une matière réfractaire suffisante.

Voici le texte original du brevet:

Notre invention a pour but de produire el d'utaliser l'énorme quantilé de chaleur que peuvent développer une flamme oxyhydrique ou un are voltaique, employés seuls ou concurremment, quand ils lèchent contıuellement les mêmes parois de la substance à échauffer.

L'apparell ou four à l'aide duquel nous réalisons celte inventıon consiste en un bloc falt de matière ou de pâte réfractaire, dans Iequel nous réservons deux, quatre ou un nombre quelconque de canaux.

St le four de haule température est à flamme oxyhydrique, chaque parre de canaux reçoit deux lubes à gaz pour l'accès de l'hydrogène et de l'oxygène dans des proportions connues.

Si l'appareil pst à arc voltaique, ces canaux reçoivent des bagueltes de charbon terminant les fils ou câhles d'une machne productive d'électricité. Ces bagueltes seront mues par des contrepoids ou par des ressorts. Elles ont leurs extrétnités noyées dans la matière réfractaire, el larc passe par deux orıfices qui les mainliennent.

On peut employer les deux sources de chaleur concurremment. Quant a la nature du bloc, il sulfil que celui-ci soit formé d'une matière réfraclaire; à cet effet, nous employons de préférence, quoıque non exclusıvement, un agglomére de magnésı calcınée et d'un oxyde mélallique, comprimés à haute pression.

La forme intérieure du four dépend du corps que l'on veut soumeltre à la haute température obtenue. C'est généralement une simple cavilé sphérique, sur laquelle on peul disposer un couvercle mobile.

Dans certains cas, ce couvercle peul être fixe, ou être percé d'une ouverture pour l'introduction des matières à traiter.

On volt fachlment que, par la disposilion indiquée, la matière à trailer sera toujours léchée par la flamme ou par l'arc, dont la position est uniformément invariable.

Les applications de ce four sont nombreuses.
Le disposılif dans lequel on fail usage d'un arc voltaïque, permettant la transtormalion du charbon en graphile, peut être ulılısé dans beaucoup de cas où celte transformation de charbon en graphite peut ander à l'actıon physique on chimrque que l'on a en vue.

La figure 1 est une coupe verticale, et la figure 3 une vue horizontale d'une première disposilion.

La figure 2 est une cuupe verticale, et la figure 4 une vue horizontale d'une deuxieme disposition.
Fig. 1 .
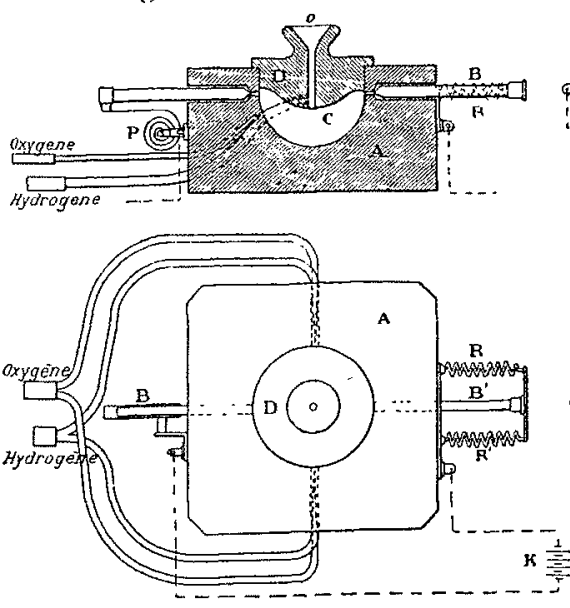

Fig. 2 .
Fig. 3.
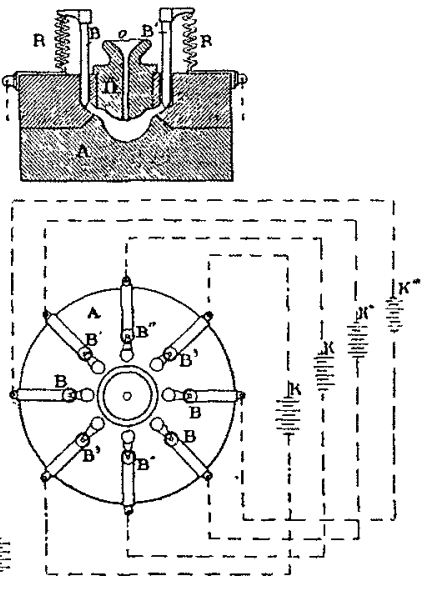

Fig. 4 .
Dans ces figures, les mêmes lettres désignent les mêmes objets : A représente le bloc fait de pâte ou de substance réfractaire; il peut être formé d'une plèce ou de plusieurs pièces frettées ; C représente la cavité qui y est ménagèe; ses dimensions varient avec le but à atteindre; $D$ représente le couvercle qui permet de maintenir la chaleur localisee dans le four, lorsqu'on veut atleindre les lempèralures les plus élevées; il est percé d'une ou de plusieurs ouvertures 0 , pour l'introduction des matières à traiter, ou l'échappement des gaz ou des vapeurs.

Si le foup est électrique, la fusion des substances traitées s'oblient au moyen d'un arc voltaîque qui se produit entre les paires de charbons BB, B'B'. B"B", elc, ayant leurs extrémités noyées dans le bloc réfractare el passant par des orifices 00'. La section de ces charbons est de forme crrculaire, mais il pourra étre avantageux de donner une section différente, polygonale, annulare ou mixte. La section de ces charbons pouvant étre très grande, on peut mélanger dans leur masse, ou leut adjoindre intérieurement des matıères destınées à augmenter leur durée. ou faciliter leur bon fonctionnement. Les crayons creux peuvent servir a introduire les jets de gaz oxyhydrique. L'avancement des charbons ou crayons peut être oblenu au moyen d'un système de ressorts, soit en spırale, comme on le volt en $P$ dans la figure 1 , ou à boudin $R, R^{\prime}$, soit en sunples lames. Ces ressorts peuvent être remplacés par des contrepoids.

Le courant peul étre produit par une ou plusieurs piles, ou par une ou plusieurs machines magnéto ou dynamo-électriques $\mathrm{K}, \mathrm{K}$, elc,, ou par une source queloonque d'électricité.

Si le four est oxyhydrique, l'oxygène et l'hydrogène sont envoyés à l'intérieur du four par une ou plusieurs paires de lubes qui amènent les gaz de réservorrs quelconques, dans les proportions convenables pour oblenir le maximum de chaleur (tig. 1 el 2).

Quel que soil le nombre de paires de câbles électriques ou de tubes à gaz, les uns et les autres doivent êtres disposès symétriquement par rapport au centre de lappareil, pour assurer l'échauffement régulier el rapide de la substance sur laquelle on opère.

En résuıné, nous revendıquons, comme étant notre propriété exclusive :

10 Les dispositions spéciales, gràce auxquelles la haute chaleur nécessaire à l'échaufrement des substances s'oblıenl; soit au moyen d'une ou de plusieurs paires de charbons ou crayons, tels qu'ils sont indrqués plus haut, recevant le courant d'une ou de plusieurs batleriea, ou sources quelconque d'électricité, d'une ou de plusieurs machines dynamos ou magnélo-électrıques, soit au moyen d'une ou plusienrs paires de lubes à gaz hydrogène et oxygène provenant de deux ou plusieurs gazomètres;

$2^{\circ}$ En général, la construction du four lel qu'il est ci dessus décrit et dessiné, formé d'une substance quelconque, mais plus 
spécialement d’un agglomére le magnésie calcinée el flun oxyde métallique, comprimes à haute pression. solt en un seul bloc soit en plusieurs plèces susceptubles d'être juxtaposées en entermées rlans une enveloppe commune, sort en fonte ou en lòle, et dans leçuel l'arc ou la flamme lèche contınuellement la subslance à traiter et la matière réfractaire du four,

$3^{0}$ Dans le cas d'un four où la haute tempéralure est déterminée par la production d'un ou de plusieurs ares voltaiques, la combinaison d'un système d'avancement des charbons, solt parallèles, soit perpendiculaires, soll obliques entre eux, à mesure de leur usure, au moyen de contrepoids ou de ressorts appropriés.

\section{CONTROLE DES DISTRIBUTIONS D'ÉNERBIE ÉLEGTRIQUE}

Le ministre des travaux publics, des postes et des télégraphes,

Vu la loi du 5 juin 1906 .

Vu le décret du 17 octobre 1907 , et notament, l'article 5 ainsi conçu :

« Les agents désignés par les municipalités pour le contrôle des distributions établies en vertu de concessions données par les communes et les syndicats de communes, et des distributions empruntant exclusivement les voies vicinales et urbaines, doivent remplir les conditions de capacité fivées par le ministre des travaux publics $)$;

Sur la proposition du directeur du personnel et de la comftabilité,

\section{Arrête :}

Article premiar. - Nul ne peut être désigné, à titre définitif, par une municipalité, pour être aff cté au contrôle des distributions d'énergie électrique, s'il n'a préalablement obtenu un certificat d'aptitude délivré par le ministre des travaux publics, des postes et des télégraphes, à la suite d'épreuves portant sur les matières définies à l'artıcle 5 ci-après:

Art. 2. - Les épreuves ont lieu lorsque les besoins du service l'exigent, et aux dates fixées par le ministre. Des avis insérées au Journal officiel font connaître ces dates en temps utile.

Art. 3. - Pour être admis à subir les épreuves, les candidats doivent être Français et âgés de vingt et un ans au ${ }^{\text {er }}$ janvier de l'année dans laquelle ont lieu ces épreuves.

Art. 4. - Les demandes d'admission aux épreuves doivent être adressées, sur papier timbré, au ministre des travaux publics, par l'internédiaire du préfet du département où résident les candidats. Elles sont accompagnées :

$I^{\circ}$ Dune expédition authentique de l'acte de naissance du candidat er, s'il y a lieu, d'un certificat établissant qu'il possède la qualité de Français ;

$2^{\circ}$ D'un certificat de moralité délivré par le maire du lieu de la résidence, ou par le commissaire de police du quartier, et dûment légalisé;

$3^{\circ}$ D'un extrait du casier judiciaire remontant au moins à six mois de date.

Les candidats appartenant déjà à une administration publique n'auront pas à produire ces pièces, mais leur demande d'admission devra être être appuyée par leurs chefs hiérarchiques.

Le ministre fait connaître aux candidats, par lettres individuelles, s'lls sont ou non admis a prendre partaux épreuves Il leur indique en même temps la ville où ils auront à se piésenter pour les subir.

Art 5. - Les épreuves sont écrites et réglées ainsi qu'il suit:

Q Questions techniques ou administratives. Tempé Coeficient tratives. 4 heures $2^{\circ}$ Rapport sur une affaire de service. ... 3 heures

Totaux...

$$
\text { . } 7 \text { heures }
$$

\section{2}

I

Art. 6. - Les épreuves ont lieu sur le programme suivant:

Electricité statique: distribution de l'électricite sur les corps; influence; potentiel; machines électro-statiques; condensation de l'électricité; électricité almosphérique.
Electricité dynamique: courants électriques; loi d'Ohm; courants dérives; duverses espèces des piles; action calorithques des courants; actions chimiques; accumlateurs.

Magnetisme: principes généraux; influence; prozédés d'aimentation; magnétisme terrestre.

Electro-magnétisme et électro-dynamique: mouvements résultant de l'action des courants sur les aimants, principes génćraux de l'électro-dynamique; mouvements résultant de l'action des courants sur les courants; action de la terre sur les courants; assimilation des courants et des aimants; aimen. tation pdíles courants

Induction: phénomènes généraux; self-induction; courants de Foucault; bobine de Ruhmkorff.

Mesures électriques: unités; mesure des intensités, galvanomètres; ampèremètres; électro-dynamomètres; mesure des différences de potentiel; voltmètres: mesure des resistances; mesure de la capacité électrique; mesure de l'énergie électrique; waumètres, compteurs; mesures magnétıques.

Production industrielle des courants; machines à courant continu, à courants alternatifs monophasés ou polyphasés.

Distribution de l'électricité : nature des conducteurs; lignes aériennes ou souterraines; section des conducteurs; isolement des canalisations; stations centrales; distributions par courant continu ou alternatifs; iransformateurs.

Ecloirage électrique : arc électrique; éclairage par incantes. cence; installation de l'éclairage électrique.

Trastion électrique: travail à développer; fonctionnement des moteurs; géneration de l'énergie; systèmes de distribuliun; lignes d'alimentation; conducteurs au niveau de la voie; conducteur aérien; prise de contact; ligne en caniveau; ligne à conducteurs sectionnés; courants polyphasés.

Précautions à prendre contre les dangers pouvant résulter des courants électriques industriels: accidents de personnes; dangers d'iucendie; phénomènes d'électrolyse; perturbations sur les communications télégraphiques ou téléphoniques; contrôle des distributions d'énergie électrique (loi du is juin rgo6 et règlements pris pour son exécution).

Art. 7. - Pour arriver à une appréciation exacte des connaissances des candidats, il est attribué à chaque partie des épreuves une valeur numérique exprimée par des chiffres variont de o à 20. Chacune des notes est multipliée par le coefficient représentant la valeur relative de la partie du programme à laquelle elle se rapporte; la somme des produits donne le nombre total des points obtenus.

Nul ne peut recevoir le certificat d'aptitude s'il n'a obtenu les deux tiers du maximum pour l'ensemble des épreuves.

Art. 8. - Une commission cen'rale, nommée par le ministre, et comprenant les fonctionnaires de l'Etat et des villes, choisit les sujets des compositions et procède à leur correction.

Les épreuves s'ouvrent simultanément dans tous les centres d'examens désıgnés par le ministre.

Dans chaque centre, il est institué par le ministre une commission qui est chargée de surveiller les épreuves.

Les sujets des compositions sont les mêmes pour toute la France; ils sont envoyés par l'administration au président de chaque commission, sous enveloppes cachetées, qui sont ouvertes, en présence des candidats, au moment fixé pour chaque épreuve. Après l'achèvement des épreuves, le président transmet à la commission centrale, par l'intermédiairc du minis. tre, toutes les compositions.

Lorsque les corrections sont terminées, la commission centrale dresse et remet au ministre la liste des candidats susceptibles de recevoir le certificat d'aptitude; celuj-ci est dólivré par le ministre des Travaux publics.

Art. 9. - Les candidats n'ont à leur disposition, pendant la durée des épreuves, ni livres, ni brochures, ni notes d'aucune sorte. Au cours des séances, ils ne peuvent avoir aucune communication avec le dehors ou entre eux.

Toute traude, dûment constatée, donne lieu à la radiation du candidat par le ministre, sans préjudice des mesures qui peú 